intensity of pain and (iii) explore associations with medical, parental and socioeconomic factors.

Methods The study was cross-sectional. Participants were parents of non-verbal CP outpatients at University Malaya Medical Centre, Kuala Lumpur and two centres in the surrounding area. Patients were aged 2-19 years. Written informed consent was obtained. The Caregiver Priorities and Child Health Index of Life with Disabilities (CPCHILDC) Questionnaire and a separate proforma were utilised for assessment of frequency and intensity of pain during the preceeding 4 weeks. Parental psychosocial well being was assessed via the Depression, Anxiety and Stress Scale (DASS-21) and the Multidimensional Scale of Perceived Social Support (MSPSS).

Results The response rate was 94\%, including 104 children (54 boys, 50 girls). The majority (51\%) had GMFCS level V and $65 \%$ had spastic quadriplegia. Reported pain was $65 \%$. The most frequent and intense pain was during physiotherapy. Nineteen $(18 \%)$ reported that their children experienced pain $>1$ time per day and $10 \%$ reported daily pain. Severe pain was reported by $17 \%$. There were no significant factors associated with frequency of pain. Older children $(p<0.05)$, those with spastic quadriplegia $(\mathrm{p}<0.05)$ and poorer quality of life $(\mathrm{p}<$ 0.05 ) had more intense pain. GMFCS level, gender, co-morbidities, parental psychosocial scores, parental education and income were not significant modifying factors of intensity of pain.

Conclusion Recognition of pain in non-verbal CP patients is crucial for holistic management.

\section{PO-0841 WITHDRAWN}

\section{PO-0842 OPTIMAL EMOTIONAL DEVELOPMENT IN YOUNG CHILDREN: THE ROLE OF PARENTS AND PAEDIATRICIANS}

SU Johnson. Discipline of Paediatrics and Child Health, University of Sydney Medical School and Children's Hospital Westmead, Sydney, Australia

10.1136/archdischild-2014-307384.1471

Background Parents and paediatricians in partnership have a unique role in influencing the emotional development of young children. The opportunity presents when parents consult paediatricians about their child's behaviour or developmental problems.

Discussion Infants and young children learn by interacting with and reacting to their environment. Early relationships with their parents foster feelings of trust and security, which influences their relationships with others as they develop and mature.

Paediatricians, through their knowledge of child development, are in a unique position to influence and guide parents in early child-rearing practices. They do newborn examinations and they assess young children through various stages of early development, preschool and school entry. They are often the point of contact in later transition periods such as high school entry and adolescence, particularly when behaviour becomes challenging.

The presentation will touch on theories of development, early experiences, resilience and cultural factors that influence emotional development. It will provide a clinician's perspective on the management of behaviour difficulties and the partnership with parents in order to facilitate optimal emotional development in young children.
Conclusion Paediatricians have an opportunity to influence healthy and positive relationships between parents and their young children that will allow children to develop to the best of their ability and become responsible members of society in the long-term.

\section{PO-0843 EFFECT OF CEREBRAL PALSIED CHILDRENS' SITTING POSTURE ON MOTHERS' QUALITY OF LIFE}

B Aras, O Aras, G Kallem. School of Health Sciences, Department of Physiotherapy, Dumlupinar University, Kütahya, Turkey

\subsection{6/archdischild-2014-307384.1472}

The aim of this study was to investigate the relation between sitting posture of diplegic spastic cerebral palsied children and the quality of life of their mothers. Twenty two children with the diagnosis of diplegic spastic cerebral palsy and their mothers were included in the study. The functional level of children were classified according to the Gross Motor Function Classification System (GMFMCs). Sitting Assessment Scale (SAS) was used to evaluate sitting posture. Nottingham Health Profile (NHP) was used to assess the quality of life of mothers. Spearman correlation analysis was used to investigate the relation between sitting posture of children and quality of life of mothers. The mean age of children and mothers were $6.3 \pm 4.6$ years, $35.7 \pm 8.6$ years, respectively. The median GMFMCs level was 4 . The mean SAS score was $13.2 \pm 3.4$ in children and, mean NHP score was $164.2 \pm 106.7$ in mothers. There was a significant correlation between SAS score and NHP score (rho=-0,622, p < 0.05). Proper sitting posture is important for feeding, carrying and caring of a child. Improvements in sitting posture of children with cerebral palsy may have favourable effects on mothers' and caregivers' quality of life.

\section{PO-0844 ASYMMETRICAL INTRAUTERINE GROWTH RESTRICTION AND LANGUAGE: WHY IS THIS IMPORTANT?}

${ }^{1} Z$ Kolundzic, ${ }^{2} \mathrm{M}$ Lencek, ${ }^{1}$ A Simic Klaric. ${ }^{1}$ Pediatric, County Hospital, Pozega, Croatia, ${ }^{2}$ Pediatric, Faculty of Education and Rehabilitation Sciences, Zagreb, Croatia

\subsection{6/archdischild-2014-307384.1473}

Neurodevelopmental outcome of children born after asymmetrical IUGR is impaired in great amounts of cases. Long term consequences of IUGR deserve special attention, because they could cause poorer cognitive and language abilities, learning difficulties and lower academic level achievement.

There is a small number of data related to language development of children born after IUGR. With regard to the Croatian language there was no research on morphological knowledge for this children, although it is known that linguistic knowledge is important for reading, writing and later school success.

Therefore the goal of this paper is to determine the level of language knowledge, especially morphological knowledge and the correlation between morphological and other linguistic variables in 40 children born after IUGR and their control peers. At the time of examination all 80 children were aged between six and seven years, average cognitive abilities.

Results showed statistically significant differences between children born after IUGR and their control peers on all language tasks, particulary in morphological items: children born after IUGR were significantly poorer. There was significantly higher correlation between morphological abilities and other language 
abilities in children born after IUGR. It seems that biological determinants may be the reason for that stronger dependence of morphological variables on other language abilities and for the formation of a compensatory language mechanisms. Children born after IUGR are risk for difficulties in reading and writing according to the structure of their linguistic knowledge: it is important for all professionals involved in follow-up process of this children.

\section{PO-0845 ACUTE NECROTISING ENCEPHOLOPATHY IN CHILDHOOD - EPIDEMIOLOGY, RADIOLOGICAL FINDINGS AND OUTCOMES}

${ }^{1} \mathrm{HY}$ Lim, ${ }^{1} \mathrm{~V}$ Ho, ${ }^{2} \mathrm{~T}$ Thomas, ${ }^{2}$ WS Chan. ${ }^{1}$ Department of Paediatric Medicine, KK Women and Children Hospital Singapore, Singapore, Singapore; ${ }^{2}$ Department of Paediatric Subspecialities, KK Women and Children Hospital Singapore, Singapore, Singapore

\subsection{6/archdischild-2014-307384.1474}

Background and aims Acute necrotizing encephalopathy in childhood (ANEC) is a disease characterised by acute encephalopathy and radiological features of bilateral thalamic necrosis. Medium and long term morbidity is not well described. We describe the mortality and morbidity outcomes in our paediatric cohort with this disease.

Methods This is a retrospective ten-year series. Children aged one month to 18 years diagnosed with 'ANEC' were collated from Neurology and Radiology databases.

18 fulfilled clinical criteria of acute encephalopathy. All were scored with Mizuguchi's radiological checklist by two paediatric neurologists and one radiologist. 11 cases scored unlikely were excluded.

Data analysis focused on discharge and follow-up outcomes. Results 7 patients were analysed. The median age was 3.7 years. All were previously well with normal development. All had impaired consciousness at presentation with preceding fever and prodrome. Typical radiology showed bilateral thalamic involvement with/without areas of haemorrhage and necrosis. Causative organisms included Influenza A H1N1, Human Herpes Virus 6 and Metapneumovirus. All were treated with steroids, immunoglobulin or both.

Outcomes were evaluated at discharge and follow-up and divided into good or poor (including death). One passed away from brainstem death. All had neurological deficit at discharge: 50\% mildly affected; 50\% severely affected. $00 \%$ in the former group restored normal neurological function on follow-up. In the latter, two responded well to rehabilitation but one remained severely impaired.

Conclusions ANE mortality at our institution is 14\%. Morbidity of survivors at discharge is $100 \%$. Long term follow up morbidity however, improves to $50 \%$ with half achieving normal neurological function at follow up.

\section{P0-0846 TUMEFACTIVE DEMYELINATING LESIONS IN JUVENILE-ONSET MULTIPLE SCLEROSIS}

'S Menascu, ${ }^{2}$ S Miron, ${ }^{3}$ A Fatal, ${ }^{2} \mathrm{~A}$ Achiron. ${ }^{1}$ Pediatric Neurology Unite Dana Children's Hospital and Multiple Sclerosis Center, Tel Aviv Medical Center and Sheba Medical Center, Tel-Aviv, Israel; ${ }^{2}$ Multiple Sclerosis Center, Sheba Medical Center, Tel-Aviv, Israel; ${ }^{3}$ Pediatric Neurology Unite Dana Children's Hospital, Tel- Aviv Medical Center, Tel-Aviv, Israel

10.1136/archdischild-2014-307384.1475

Background The pathogenesis of large demyelinating lesions is still controversial. Atypical tumefactive demyelinating lesions
(TDL) associated with acute inflammation, peri-lesional oedema and gadolinium ring enhancement are infrequently described in patients with juvenile-onset multiple sclerosis (MS).

Objective To describe the clinical, imaging and micro-structural metrics of TDLs and chronic MS lesions in patients with juvenile-onset MS.

Methods Ten patients diagnosed with MS were analysed for the presence of TDLs and chronic non enhancing MS lesions. The MS lesions were defined by a region of interest encircling the lesion centre on 2-3 consecutive slices. DTI images were acquired along 31 independent orientations using a single shot echo-planar imaging sequence.

Results Four patients with 6 TDL, developed acute neurological symptomatology. The two girls presented with acute ataxia and aphasia, and the two boys with severe ataxia. Three patients progressed rapidly to develop seizures, became stuporotic and were admitted to the paediatric intensive care unit. Brain MRI demonstrated six TDLs. Analysis of the whole group (10 patients) disclosed 21 chronic non enhancing lesions. Assessment of DTI metrics of TDL as compared to chronic MS lesions disclosed significant differences.

Conclusion TDL are a possible presentation of demyelinating disorders, posing a diagnostic and therapeutic dilemma towards neoplastic lesions. The micro-structural analysis of TDL suggests a severe tissue disruption probably due to the acute inflammatory process and oedema. Our analysis provides metrical tools that together with MR spectroscopy and perfusion may aid to identify accurately TDLs, potentially sparing young patients unnecessary and possibly debilitating brain biopsy.

\section{PO-0847 WITHDRAWN}

\section{PO-0848 WITHDRAWN}

\section{PO-0849 EXPRESSION PATTERN OF BRAIN SPECIFIC MIR-124, MIR-134, AND MIR-9 IN AN IMMATURE RAT MODEL AND CHILDREN WITH MESIAL TEMPORAL LOBE EPILEPSY}

A Omran, S El Sharkawy. Pediatrics and Neonatology, Faculty of Medicine Suez Canal University, Ismailia, Egypt

\subsection{6/archdischild-2014-307384.1476}

Background and aims Mesial temporal lobe epilepsy (MTLE) is a particularly devastating form of human epilepsy with significant incidence of medical intractability. MicroRNAs (miRs) are small, noncoding RNAs that regulate post-transcriptional expression of protein-coding mRNAs, which may have key roles in the pathogenesis of MTLE development. We aimed to detect the dynamic expression pattern of brain specific miR-124, miR-134 and miR-9 in the hippocampi of immature rats and children with MTLE.

Methods To study the dynamic expression pattern of brain specific miR-124, miR-134 and miR-9, we performed real-time quantitative PCR on the hippocampi of immature rats at 25 days of age. Expression was monitored in the acute, latent, and chronic stages of disease $(2 \mathrm{~h}$ and 3 and 8 weeks after induction of lithium-pilocarpine status epilepticus, respectively), and in control hippocampal tissues corresponding to the same 